astro-ph/0503023

CERN-PH-TH/2005-030

UMN-TH-2345/05

FTPI-MINN-05/04

\title{
The Effects of Unstable Particles on Light-Element Abundances: Lithium versus Deuterium and ${ }^{3} \mathrm{He}$
}

\author{
John Ellis ${ }^{1}$, Keith A. Olive ${ }^{2}$ and Elisabeth Vangioni ${ }^{3}$ \\ ${ }^{1}$ TH Division, Physics Department, CERN, CH-1211 Geneva 23, Switzerland \\ ${ }^{2}$ Theoretical Physics Institute, University of Minnesota, Minneapolis, MN 55455, USA \\ ${ }^{3}$ Institut d'Astrophysique de Paris, F-75014 Paris, France
}

\begin{abstract}
We reconsider the effects of unstable particles on the production and destruction of the primordial light elements, with a view to reconciling the high primordial ${ }^{7} \mathrm{Li}$ abundance deduced from Big Bang Nucleosynthesis (BBN), as implied by the baryon-to-photon ratio now inferred from the anisotropies of the Cosmic Microwave Background (CMB), with the lower abundance of ${ }^{7} \mathrm{Li}$ observed in halo stars. The potential destruction of ${ }^{7} \mathrm{Li}$ is strongly constrained by observations of Deuterium (D), ${ }^{3} \mathrm{He}$ and ${ }^{6} \mathrm{Li}$. We identify ranges for the unstable particle abundance and lifetime which would deplete ${ }^{7} \mathrm{Li}$ while remaining consistent with the abundance of ${ }^{6} \mathrm{Li}$. However, in these regions either the $\mathrm{D}$ abundance is unacceptably low or the ratio ${ }^{3} \mathrm{He} / \mathrm{D}$ is unacceptably large. We conclude that late particle decay is unable to explain both the discrepancy of the calculated ${ }^{7} \mathrm{Li}$ abundance and the observed ${ }^{7} \mathrm{Li}$ plateau. In the context of supersymmetric theories with neutralino or gravitino dark matter, we display the corresponding light-element constraints on the model parameters.
\end{abstract}

CERN-PH-TH/2005-030 


\section{Introduction}

The observed abundances of light elements are generally in good agreement with the predictions of Big Bang Nucleosynthesis (BBN) calculated assuming a homogeneous RobertsonWalker-Friedman cosmology [1]. Within this framework, the success of BBN calculations imposes important constraints on the number of light particle species and on the baryon-tophoton ratio $\eta$ [2]. Recent high-precision measurements of the cosmic microwave background (CMB) radiation by WMAP and other experiments now complement the BBN in important ways [3]. For example, they impose strong constraints on $\eta$ and weaker constraints on the number of light particle species. Of particular interest are the very precise predictions of the light element abundances from BBN that are made with the CMB value of $\eta$ [4].

Based on these predictions, there is now tension between some observed light-element abundances and those that would be calculated using the CMB value of $\eta$ and assuming no additional light particles beyond three light neutrino species. In particular, the prediction for the primordial abundance of ${ }^{7} \mathrm{Li}$ made using the $\mathrm{CMB}$ value of $\eta[5,6,7,8,9]$ is somewhat higher (by a factor of 2 - 3) than the primordial abundance inferred from astrophysical observations. The significance of this discrepancy should not be over-emphasized, in view of the potential systematic errors in the interpretation of the astrophysical data. However, it has stimulated theoretical explorations of mechanisms for modifying the CMB/BBN prediction for ${ }^{7} \mathrm{Li}$, for example via the late decays of massive particles $[10,11]$.

The effects of such decays have been studied extensively, and the constraints imposed on them by the observed abundances of ${ }^{4} \mathrm{He}$, Deuterium and ${ }^{6} \mathrm{Li}$ are well understood $[12,13,14]$. Previous studies had shown no incompatibilities between these constraints and the suggestion that late-decaying particles might have modified the BBN prediction for the abundance of ${ }^{7} \mathrm{Li}$ so as to agree better with the astrophysical observations. Supersymmetric models with conserved $R$ parity naturally predict such a particle, either the gravitino if it is not the lightest supersymmetric particle (LSP), or a neutralino or stau slepton if the gravitino is the LSP $[15,16]$, which may well have the appropriate abundance and lifetime to affect both the ${ }^{6} \mathrm{Li}$ and ${ }^{7} \mathrm{Li}$ abundances. Later in this paper, we identify these regions in the parameter space of a constrained version of the minimal supersymetric extension of the Standard Model (CMSSM), as well as in very constrained versions of the model [17] motivated by supergravity considerations, which predict a gravitino LSP in parts of the parameter space.

However, we also point out in this paper that such scenarios typically yield an abundance of ${ }^{3} \mathrm{He}$ that may be more than an order of magnitude larger than the Deuterium abundance. Since it appears that the abundance of ${ }^{3} \mathrm{He}$ has remained relatively constant in time, whereas Deuterium (D) would have been destroyed in stars, it seems unlikely that the ratio of the ${ }^{3} \mathrm{He}$ to $\mathrm{D}$ abundances could have been significantly larger than it present value in the early history of the Universe [14]. Imposing this constraint, we find a further restriction on the parameter space of late-decaying heavy-particle models, which excludes the region of parameter space where they could have the desired impact on the primordial abundance of ${ }^{7} \mathrm{Li}$. We display this and other constraints on the lifetime and abundance of any massive unstable relic particle such as a gravitino, pointing out also the potential impact of weakening the lower limit on the

primordial Deuterium abundance. We also display the effects of the ${ }^{3} \mathrm{He}$ constraint on the 
parameter spaces of models in which heavier supersymmetric sparticles decay into gravitinos. The overall reductions in the allowed parameter spaces are often small, but they do exclude the regions where the abundance of ${ }^{7} \mathrm{Li}$ could be brought into line with the astrophysical observations. This may motivate a reassessment of their interpretation.

\section{Is there a problem with Lithium-7?}

The most direct and accurate estimate of the baryon-to-photon ratio $\eta$ is currently provided by the acoustic structures in the CMB perturbations [3], namely

$$
\eta=6.14 \pm 0.25 \times 10^{-10}
$$

This range may be used an input into homogeneous BBN calculations [5, 6, 7, 8, 9], yielding the following abundances for the elements of principal interest, which are taken from [9]:

$$
\begin{aligned}
Y_{p} & =0.2485 \pm 0.0005, \\
\frac{\mathrm{D}}{\mathrm{H}} & =2.55_{-0.20}^{+0.21} \times 10^{-5}, \\
\frac{{ }^{3} \mathrm{He}}{\mathrm{H}} & =1.01 \pm 0.07 \times 10^{-5}, \\
\frac{{ }^{7} \mathrm{Li}}{\mathrm{H}} & =4.26_{-0.60}^{+0.73} \times 10^{-10}, \\
\frac{{ }^{6} \mathrm{Li}}{\mathrm{H}} & =1.3 \pm 0.1 \times 10^{-14},
\end{aligned}
$$

where $Y_{p}$ is the ${ }^{4}$ He mass fraction, and the other abundances are expressed in terms of their numbers relative to $\mathrm{H}$, as shown.

These abundances may then be compared with the abundances of the same elements that are inferred from those observed in the most primitive astrophysical sites [18, 19, 20, 21, 22]:

$$
\begin{aligned}
Y_{p} & =0.232 \text { to } 0.258 \\
\frac{\mathrm{D}}{\mathrm{H}} & =2.78 \pm 0.29 \times 10^{-5} \\
\frac{{ }^{3} \mathrm{He}}{\mathrm{H}} & =1.5 \pm 0.5 \times 10^{-5} \\
\frac{{ }^{7} \mathrm{Li}}{\mathrm{H}} & =1.23_{-0.32}^{+0.68} \times 10^{-10} \\
\frac{{ }^{6} \mathrm{Li}}{\mathrm{H}} & =6_{-3}^{+7} \times 10^{-12}
\end{aligned}
$$

Note that, for ${ }^{3} \mathrm{He}$, we have at our disposal only local data coming from star-forming HII regions in the galactic disk [23] or from the proto-solar value [24]. Comparing the two sets of abundances, (2) and (3), we see no significant discrepancies, except in the case of ${ }^{7} \mathrm{Li}$ and ${ }^{6} \mathrm{Li}$. However, whereas ${ }^{7} \mathrm{Li}$ has to be destroyed, one must produce a factor 1000 more ${ }^{6} \mathrm{Li}$. 
The value quoted above for the ${ }^{7} \mathrm{Li}$ abundance assumes that $\mathrm{Li}$ depletion is negligible in the stars observed. Indeed, standard stellar evolution models predict Li depletion factors which are very small (less than $0.05 \mathrm{dex}$ ) in very metal-poor turnoff stars [25]. However, there is no reason to believe that such simple models incorporate all effects which could lead to depletion, such as rotationally-induced mixing and/or diffusion. Including these effects, current estimates for possible depletion factors are in the range $\sim 0.2-0.4 \operatorname{dex}[26]$. However, the data sample [27] used in deriving the abundance in (3) shows a negligible intrinsic spread in $\mathrm{Li}$, leading to the conclusion that depletion in these stars is in fact quite low (less than 0.1 dex).

Another important source of potential systematic uncertainty is related to the assumed surface temperature of the star. A recent study [28] found significantly and systematically higher temperatures for stars used in ${ }^{7} \mathrm{Li}$ observations, specifically at low metallicity. This result leads to ${ }^{7} \mathrm{Li} / \mathrm{H}=(2.34 \pm 0.32) \times 10^{-10}$, which is still, however, nearly a factor of 2 smaller than the BBN/WMAP prediction. We note, finally, that another potential source for theoretical uncertainty lies in the $\mathrm{BBN}$ calculation of the ${ }^{7} \mathrm{Li}$ abundance. However, this too has been shown to be incapable of resolving the ${ }^{7} \mathrm{Li}$ discrepancy $[6,29]$.

The comparison of (2) and (3) also shows a discrepancy for ${ }^{6} \mathrm{Li}$. It has generally been assumed that ${ }^{6} \mathrm{Li}$ is produced in post-BBN processes such as galactic cosmic-ray nucleosynthesis [30]. Until recently, the abundance of ${ }^{6} \mathrm{Li}$ had been observed only in a few metal-poor halo stars with metallicity $[\mathrm{Fe} / \mathrm{H}]$ larger than -2.3 . However, new observations of this isotope have now been obtained in halo stars. New values of the ratio ${ }^{6} \mathrm{Li} /{ }^{7} \mathrm{Li}$ have been measured with UVES at the VLT-UT2 Kueyen ESO telescope, in halo stars with metallicity ranging from -2.7 to $-0.5[21,22]$. These observations indicate the presence of a plateau in ${ }^{6} \mathrm{Li} / \mathrm{H}$ $\simeq 10^{-11}$, suggesting a pregalactic origin for the formation of ${ }^{6} \mathrm{Li}$ [31]. These data provide interesting new constraints in the present context.

The relation of the observed ${ }^{7} \mathrm{Li}$ abundance to its primordial value may be debated, but for the moment we take the discrepancy between the observed and calculated ${ }^{7} \mathrm{Li}$ abundances at face value, and explore its possible theoretical interpretation in terms of late-decaying massive particles, depleting ${ }^{7} \mathrm{Li}$ and possibly producing ${ }^{6} \mathrm{Li}$, without negative effects on $\mathrm{D}$ and ${ }^{3} \mathrm{He}$.

\section{$3 \quad$ Possible Impact of Late-Decaying Particles}

The decays of massive particles $X$ with lifetimes $>10^{2}$ s could, in principle, have modified the BBN predictions in either of two ways. First, their decay products would have increased the entropy in the primordial plasma, implying that its value during BBN was lower than that inferred from the CMB. However, this effect is negligible compared with the second effect, which is the modification of the BBN light-element abundances by the interactions of decay products [12].

The latter possibility has been explored recently as a mechanism for reducing the primordial ${ }^{7} \mathrm{Li}$ abundance $[10,11]$. The existence of such late-decaying massive particles $X$ is a generic possibility in supersymmetric models, in particular $[15,16]$. Examples include a massive gravitino weighing $\sim 100 \mathrm{GeV}$, if it is not the lightest supersymmetric particle 
(LSP), or some other next-to-lightest supersymmetric particle (NSP) if the gravitino is the LSP. Cosmological constraints on such scenarios have been explored previously $[12,13,14]$. However, the potential ${ }^{7} \mathrm{Li}$ problem motivates a re-examination of the astrophysical and cosmological constraints on such scenarios. In particular, we wish to determine whether or not a possible solution to this problem can be found in the context of motivated and well-studied supersymmetric models.

\subsection{The $\mathrm{D}$ and ${ }^{4} \mathrm{He}$ Abundances}

Fig. 1 shows the current constraints in the $\left(\tau_{X}, \zeta_{X}\right)$ plane, updating those shown in [13]. The green lines are the contours

$$
(1.3 \text { or } 2.2) \times 10^{-5}<\frac{\mathrm{D}}{\mathrm{H}}<5.3 \times 10^{-5} .
$$

The first of the lower bounds is the higher line to the left of the cleft, and represents the very conservative lower limit on $\mathrm{D} / \mathrm{H}$ assumed in [13]. The range $1.3-5.3 \times 10^{-5}$ effectively brackets all recent observations of $\mathrm{D} / \mathrm{H}$ in quasar absorption systems. The second of the lower bounds is the lower line on the left side, and represents what now seems a reasonable lower bound, which is obtained from the $2-\sigma$ lower limit in (3). The upper bound in (4) is the line to the right of the cleft, and is the same upper limit as was used in [13]. A priori, there is also a narrow strip at larger $\zeta_{X}$ and $\tau_{X}$ where the $\mathrm{D} / \mathrm{H}$ ratio also falls within the range (4), but this is excluded by the observed ${ }^{4} \mathrm{He}$ abundance.

The solid red lines in the upper right part of Fig. 1 correspond to the limits

$$
Y_{p}>0.227 \text { or } 0.232
$$

where the lower number (corresponding to the higher line) was used in [13], and the higher number (corresponding to the lower line) is a lower limit that has been advocated recently [18]. It is apparent that, for our purposes, the third significant figure in the ${ }^{4} \mathrm{He}$ abundance is unimportant: the narrow $\mathrm{D} / \mathrm{H}$ strip is in any case excluded, and there are always stronger bounds on $\zeta_{X}$ at large $\tau_{X}$.

\subsection{The ${ }^{6} \mathrm{Li}$ Abundance}

As said above, recent observations of ${ }^{6} \mathrm{Li}$ in halo stars have provided new insight into the origin and the evolution of this isotope. We recall that ${ }^{6} \mathrm{Li}$ is a pure product of spallation, and many studies have followed the evolution of ${ }^{6} \mathrm{Li}$ in our Galaxy [30]. Of particular importance in this context is the $\alpha+\alpha$ reaction that leads to the synthesis of this isotope as well as ${ }^{7} \mathrm{Li}$, and is efficient very early in the evolutionary history of the Galaxy. The new values of ${ }^{6} \mathrm{Li} /{ }^{7} \mathrm{Li}$ that have been measured in halo stars with UVES at the VLT-UT2 Kueyen ESO telescope indicate the presence of a plateau in ${ }^{6} \mathrm{Li}$, which suggests a pregalactic origin for the formation of this isotope. The evolution of ${ }^{6} \mathrm{Li}$ with redshift was calculated [31] following an initial burst of cosmological cosmic rays up to the formation of the Galaxy. This process is able to produce the required abundance of ${ }^{6} \mathrm{Li}$ observed in metal-poor halo 


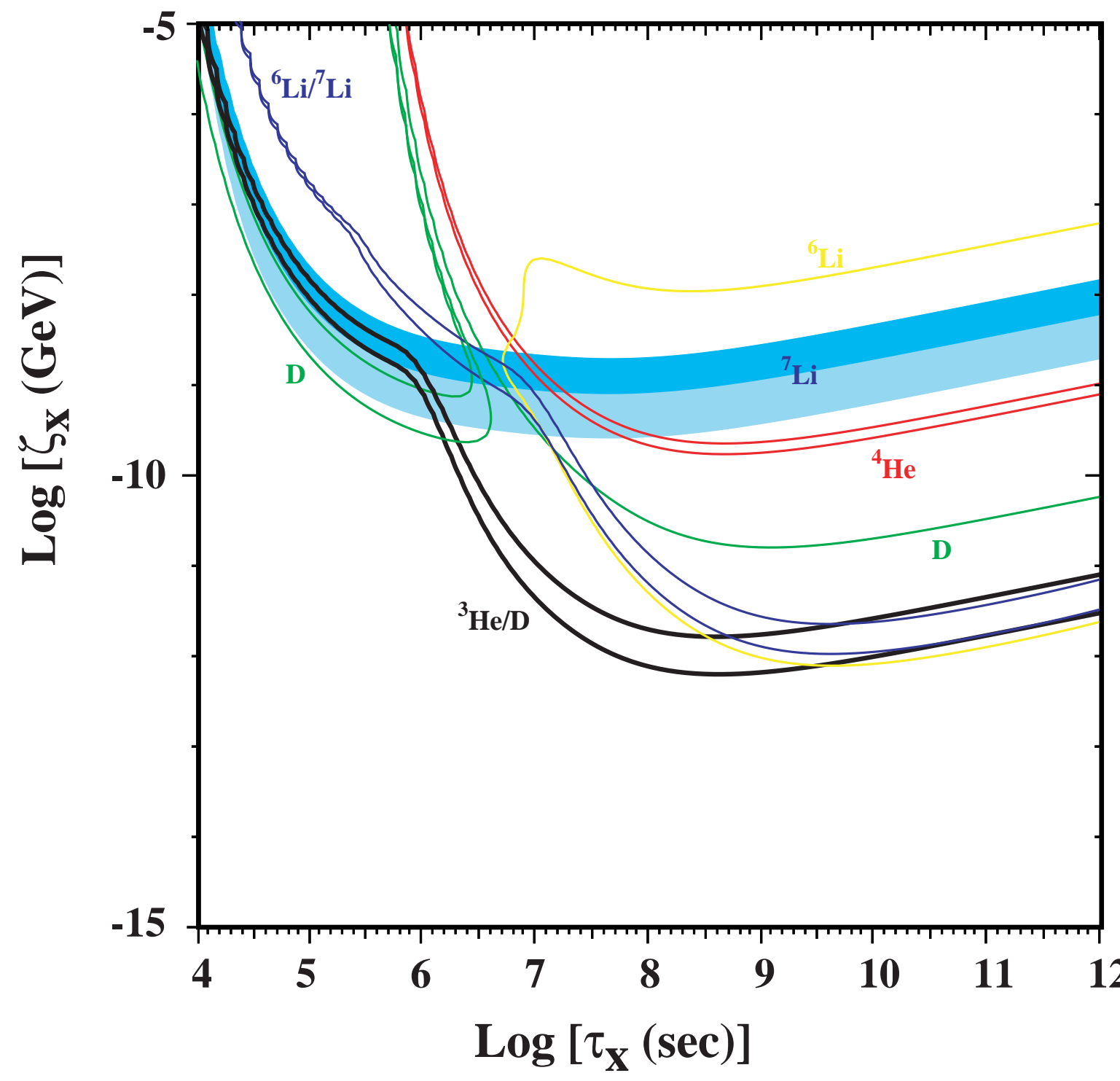

Figure 1: The constraints imposed by the astophysical observations of ${ }^{4} \mathrm{He}$ (red lines), $\mathrm{D} / \mathrm{H}$ (green lines), ${ }^{6} \mathrm{Li}$ (yellow line), ${ }^{6} \mathrm{Li} /{ }^{7} \mathrm{Li}$ (blue lines), ${ }^{7} \mathrm{Li}$ (blue band) and ${ }^{3} \mathrm{He}$ (black lines). 
stars without the additional over-production of ${ }^{7} \mathrm{Li}$. In this paper, we have to consider the new constraint brought by the existence of this plateau. The potential destruction of ${ }^{7} \mathrm{Li}$ by unstable particles must not lead to over-production of ${ }^{6} \mathrm{Li}$.

The constraint imposed by the ${ }^{6} \mathrm{Li}$ abundance is shown as a solid yellow line in Fig. 1, which is the same as that discussed in [13]. Also shown, as solid blue lines, are two contours representing possible upper limits on the ${ }^{6} \mathrm{Li} /{ }^{7} \mathrm{Li}$ ratio:

$$
\frac{{ }^{6} \mathrm{Li}}{{ }^{7} \mathrm{Li}}<0.07 \text { or } 0.15
$$

with the upper (lower) contour corresponding to the upper (lower) number in (6). The lower number was used in [13] and represented the upper limit available at the time, which was essentially based on multiple observations of a single star. The most recent data [21, 22] includes observations of several stars. The Li isotope ratio for most metal-poor stars in the sample is as high as 0.15 , and we display that upper limit here. The main new effect of this constraint is to disallow a region in the near-vertical cleft between the upper and lower limits on $\mathrm{D} / \mathrm{H}$, as seen in Fig. 1.

\subsection{The ${ }^{7} \mathrm{Li}$ Abundance}

The main region of interest in Fig. 1 is the blue shaded band that represents the inferred ${ }^{7} \mathrm{Li}$ abundance:

$$
0.9 \times 10^{-10}<\frac{{ }^{7} \mathrm{Li}}{\mathrm{H}}<(2 \text { or } 3) \times 10^{-10},
$$

with the ${ }^{7} \mathrm{Li}$ abundance decreasing as $\zeta_{X}$ increases and the intensity of the shading changing at the intermediate value. In [13], only the lower bound was used due the existing discrepancy between the primordial and observationally determined values. It is apparent that ${ }^{7} \mathrm{Li}$ abundances in the lower part of the range (7) are possible only high in the Deuterium cleft, and even then only if one uses the recent and more relaxed limit on the ${ }^{6} \mathrm{Li} /{ }^{7} \mathrm{Li}$ ratio (6). Values of the ${ }^{7} \mathrm{Li}$ abundance in the upper part of the range $(7)$ are possible, however, even if one uses the more stringent constraint on ${ }^{6} \mathrm{Li} /{ }^{7} \mathrm{Li}$. In this case, the allowed region of parameter space would also extend to lower $\tau_{X}$, if one could tolerate values of $\mathrm{D} / \mathrm{H}$ between 1.3 and $2.2 \times 10^{-5}$.

For the convenience of the subsequent discussion, the region of the $\left(\tau_{X}, \zeta_{X}\right)$ plane that is of interest for lowering the ${ }^{7} \mathrm{Li}$ abundance is shown alone in panel (a) of Fig. 2. The blue region in the arc at low $\tau_{X}$ is the region excluded by the stronger lower limit on the Deuterium abundance: $\mathrm{D} / \mathrm{H}>2.2 \times 10^{-5}$, and the red region to its right is the extra domain that is excluded by the ${ }^{3} \mathrm{He} / \mathrm{D}$ ratio, as we discuss below.

\section{The Importance of the ${ }^{3} \mathrm{He}$ Abundance}

We now come to the constraint from ${ }^{3} \mathrm{He}$, namely that the ${ }^{3} \mathrm{He} / \mathrm{D}$ ratio is absurdly high in the Deuterium cleft. Panel (b) of Fig. 2 shows a histogram of the values of the ${ }^{3} \mathrm{He} / \mathrm{D}$ ratio 

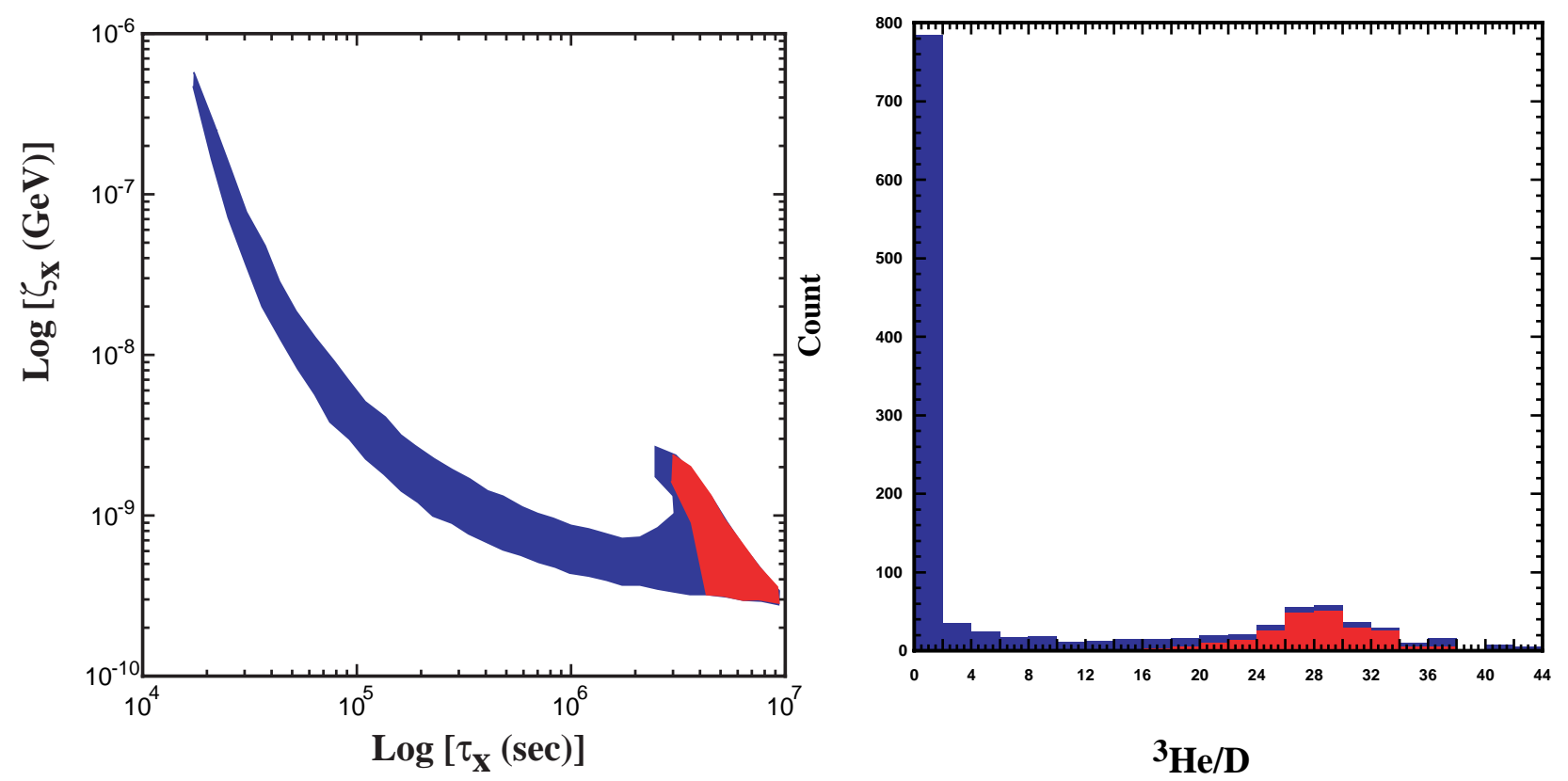

Figure 2: (a) The region of the $\left(\tau_{X}, \zeta_{X}\right)$ plane in which a decaying relic particle could have the desired impact on the ${ }^{7} \mathrm{Li}$ abundance. To derive the blue (darker grey) region, the only abundance cuts applied are: $0.9<{ }^{7} \mathrm{Li} / \mathrm{H} \times 10^{10}<3.0,1.3<\mathrm{D} / \mathrm{H} \times 10^{5}<5.3$, and ${ }^{6} \mathrm{Li} /{ }^{7} \mathrm{Li}$ $<0.15$. To obtain the red (lighter grey) region, the lower bound on $\mathrm{D} / \mathrm{H}$ was increased to $2.2 \times 10^{-5}$. (b) A histogram of the ${ }^{3} \mathrm{He} / \mathrm{D}$ ratios found in scenarios sampling the region displayed in panel (a), with similar colour coding.

found in a dense sample of scenarios in the interesting regions shown in panel (a). Since Deuterium is more fragile than ${ }^{3} \mathrm{He}$, whose abundance is thought to have remained roughly constant since primordial nucleosynthesis when comparing the BBN value to it proto-solar abundance, one would expect, in principle, the ${ }^{3} \mathrm{He} / \mathrm{D}$ ratio to have been increased by stellar processing. Indeed, there is considerable uncertainty in the evolution of ${ }^{3} \mathrm{He}$ [32]. This uncertainty is largely associated with the degree to which ${ }^{3} \mathrm{He}$ is produced or destroyed in stars. Since D is totally destroyed in stars, the ratio of ${ }^{3} \mathrm{He} / \mathrm{D}$ can only increase in time or remain constant if ${ }^{3} \mathrm{He}$ is also completely destroyed in stars. The present or proto-solar value of ${ }^{3} \mathrm{He} / \mathrm{D}$ can therefore be used to set an upper limit on the primordial value. Fig. 1 displays the upper limits

$$
\frac{{ }^{3} \mathrm{He}}{D}<1 \text { or } 2
$$

as solid black lines. Above these contours, the value of ${ }^{3} \mathrm{He} / \mathrm{D}$ increases very rapidly, and points high in the Deuterium cleft of Fig. 1 have absurdly high values of ${ }^{3} \mathrm{He} / \mathrm{D}$, exceeding the limit (8) by an order of magnitude or more. These are the red points producing the high-end peak of the histogram shown in panel (b) of Fig. 2, whereas the blue points are those excluded by the lower limit $\mathrm{D} / \mathrm{H}>2.2 \times 10^{-5}$ that is now preferred. We see that these points mostly have acceptably low values of ${ }^{3} \mathrm{He} / \mathrm{D}$, though some large values are found near the boundary with the red region in panel (a) of Fig. 2.

It is interesting to note that in the red region which has acceptable $\mathrm{D} / \mathrm{H}$ and a ${ }^{7} \mathrm{Li} / \mathrm{H}$ 
abundance low enough to match the observed values, the ${ }^{6} \mathrm{Li}$ abundance is relatively high: $7.3 \times 10^{-12}<{ }^{6} \mathrm{Li} / \mathrm{H}<1.6 \times 10^{-11}$. This matches the new ${ }^{6} \mathrm{Li}$ observations quite well, and would circumvent the need for an early period of ${ }^{6} \mathrm{Li}$ production by cosmological cosmic rays. Unfortunately, ${ }^{3} \mathrm{He} / \mathrm{D}$ ranges from $17-37$ for these parameter values.

The previous upper limit on $\eta_{X}$ [13] corresponded to the constraint $m_{X} n_{X} / n_{\gamma}<5.0 \times$ $10^{-12} \mathrm{GeV}$ for $\tau_{X}=10^{8} \mathrm{~s}$. The weaker (stronger) version of the ${ }^{3} \mathrm{He}$ constraint adopted here corresponds to

$$
m_{X} \frac{n_{X}}{n_{\gamma}}<2.0(0.8) \times 10^{-12} \mathrm{GeV}
$$

for $\tau_{X}=10^{8} \mathrm{~s}$. However, the impact of the ${ }^{3}$ He constraint is even stronger for $\tau_{X}=10^{7} \mathrm{~s}$, the location of the previous Deuterium cleft. The analysis of [13] would have given $m_{X} n_{X} / n_{\gamma}<$ $360 \times 10^{-12} \mathrm{GeV}$, whereas the weaker (stronger) ${ }^{3} \mathrm{He}$ constraint adopted here corresponds to

$$
m_{X} \frac{n_{X}}{n_{\gamma}}<9.3(3.8) \times 10^{-12} \mathrm{GeV}
$$

for $\tau_{X}=10^{7} \mathrm{~s}$.

\section{Applications to Supersymmetric Scenarios}

We now discuss some examples of the consequences of the ${ }^{3} \mathrm{He}$ constraint for various supersymmetric scenarios in which $R$ parity is conserved. In such models, if the gravitino is not the LSP it will generically decay gravitationally with a long lifetime. If the gravitino is the LSP, the next-to-lightest supersymmetric particle (NSP) will decay gravitationally into the gravitino LSP, again with a long lifetime.

\subsection{Models with an Unstable Gravitino}

We first consider the possibility that the gravitino is not the lightest supersymmetric particle (LSP), which is instead the lightest neutralino $\chi$. In this case, the gravitino is unstable, with a lifetime that could well fall within the range considered here. In such a scenario, the lightelement abundances impose an important upper limit on the possible temperature of the Universe, e.g., during reheating after inflation, which we denote by $T_{R}[12,13,14]$. We recall that thermal reactions are estimated to produce an abundance of gravitinos given by [13]:

$$
\frac{n_{m_{3 / 2}}}{n_{\gamma}}=(0.7-2.7) \times 10^{-11} \times\left(\frac{T_{R}}{10^{10} \mathrm{GeV}}\right) .
$$

Assuming that $m_{3 / 2}=100 \mathrm{GeV}$ and $\tau_{X}=10^{8} \mathrm{~s}$, and imposing the constraints (9), we now find

$$
T_{R}<(0.8-2.8) \times 10^{7} \mathrm{GeV},\left((0.3-1.1) \times 10^{7} \mathrm{GeV}\right)
$$

for the weaker (stronger) version of the ${ }^{3} \mathrm{He}$ constraint. This becomes an even more significant constraint on inflationary models, which were already somewhat embarrassed by the previous upper limit $T_{R} \sim 2 \times 10^{7} \mathrm{GeV}$. 


\subsection{Models with Gravitino Dark Matter}

We now consider the possibility that the gravitino is itself the LSP, in which case the next-tolightest supersymmetric particle (NSP) would instead be unstable, also with a long lifetime. We will work in the context of the constrained MSSM (CMSSM) [33, 34, 35], so that all the scenarios we consider have universal soft supersymmetry-breaking scalar masses $m_{0}$ and gaugino masses $m_{1 / 2}$ at the GUT scale before renormalization. The magnitude of the Higgsino mixing parameter $|\mu|$ and the pseudoscalar Higgs mass $m_{A}$ are fixed by the electroweak vacuum conditions. We consider scenarios with $\mu>0$, which are favoured by $g_{\mu}-2$ and, to a lesser extent, $b \rightarrow s \gamma$. The scenarios that we study differ in their assumptions about the relationship of the gravitino mass $m_{3 / 2}$ to $m_{0}$, but they all share the common feature that the LSP is the gravitino in generic domains of parameter space.

In the first set of scenarios, shown in Fig. 3, we fix the ratio of supersymmetric Higgs vacuum expectation values $\tan \beta=10$, which is among the lower values consistent with our hypotheses, and assume (a) $m_{3 / 2}=10 \mathrm{GeV}$, (b) $m_{3 / 2}=100 \mathrm{GeV}$, (c) $m_{3 / 2}=0.2 m_{0}$ and (d) $m_{3 / 2}=m_{0}$. In each panel of Fig. 3, we display accelerator, astrophysical and cosmological constraints in the corresponding $\left(m_{1 / 2}, m_{0}\right)$ planes, concentrating on the regions to the right of the near-vertical black lines, where the gravitino is the LSP. The vertical black dashed and (red) dot-dashed lines represent the lower limits on $m_{1 / 2}$ implied for each value of $m_{0}$ by the non-observation of a chargino and a Higgs boson at LEP, the latter having a theoretical uncertainty $\delta m_{1 / 2} \sim 50 \mathrm{GeV}$. The (pale green) narrow diagonal strips represent the regions where the relic density of the NSP would have lain in the range $0.094 \leq \Omega h^{2} \leq 0.129$ favoured by WMAP and other measurements of the cold dark matter density, if the gravitino had not been the LSP. In fact, the gravitino is always the LSP in the scenarios considered. The NSP may be either the lightest neutralino $\chi$ or the lighter supersymmetric partner of the $\tau$ lepton: this is lighter than the neutralino $\chi$ below the (red) dotted line.

Below and to the right of the upper (purple) dashed lines, the density of relic gravitinos produced in the decays of other supersymmetric particles is always below the WMAP upper limit: $\Omega_{3 / 2} h^{2} \leq 0.129$. To the right of the lower black solid lines, the lifetime of the nextto-lightest supersymmetric particle (NSP) falls below $10^{4} \mathrm{~s}$, and the analysis of [13] cannot evaluate the astrophysical constraints from the light-element abundances, in the absence of a suitably modified BBN code. The code used in [13], when combined with the observational constraints used in [13], yielded the astrophysical constraint represented by the dashed greygreen lines in the different panels of Fig. 3.

These constraints on the CMSSM parameter plane were computed in [16]. For each point in the $\left(m_{1 / 2}, m_{0}\right)$, the relic density of either $\chi$ or $\tilde{\tau}$ is computed and $\zeta_{X}$ is determined using $\Omega_{X} h^{2}=3.9 \times 10^{7} \mathrm{GeV} \zeta_{X}$. When $X=\tilde{\tau}, \zeta_{X}$ is reduced by a factor of 0.3 , as only $30 \%$ of stau decays result in electromagnetic showers which affect the element abundances at these lifetimes. In addition, at each point, the lifetime of the NSP is computed. Then for each $\tau_{X}$, the limit on $\zeta_{X}$ is found from the results shown in Fig. 1. The region to the right of this curve where $r=\zeta_{X} / \zeta_{X}^{\text {limit }}<1$ is allowed.

The astrophysical constraints obtained with the newer abundance limits used here yields the solid red lines in Fig. 3. The examples where $\tau_{X}$ and $\zeta_{X}$ for the NSP decays fall within 

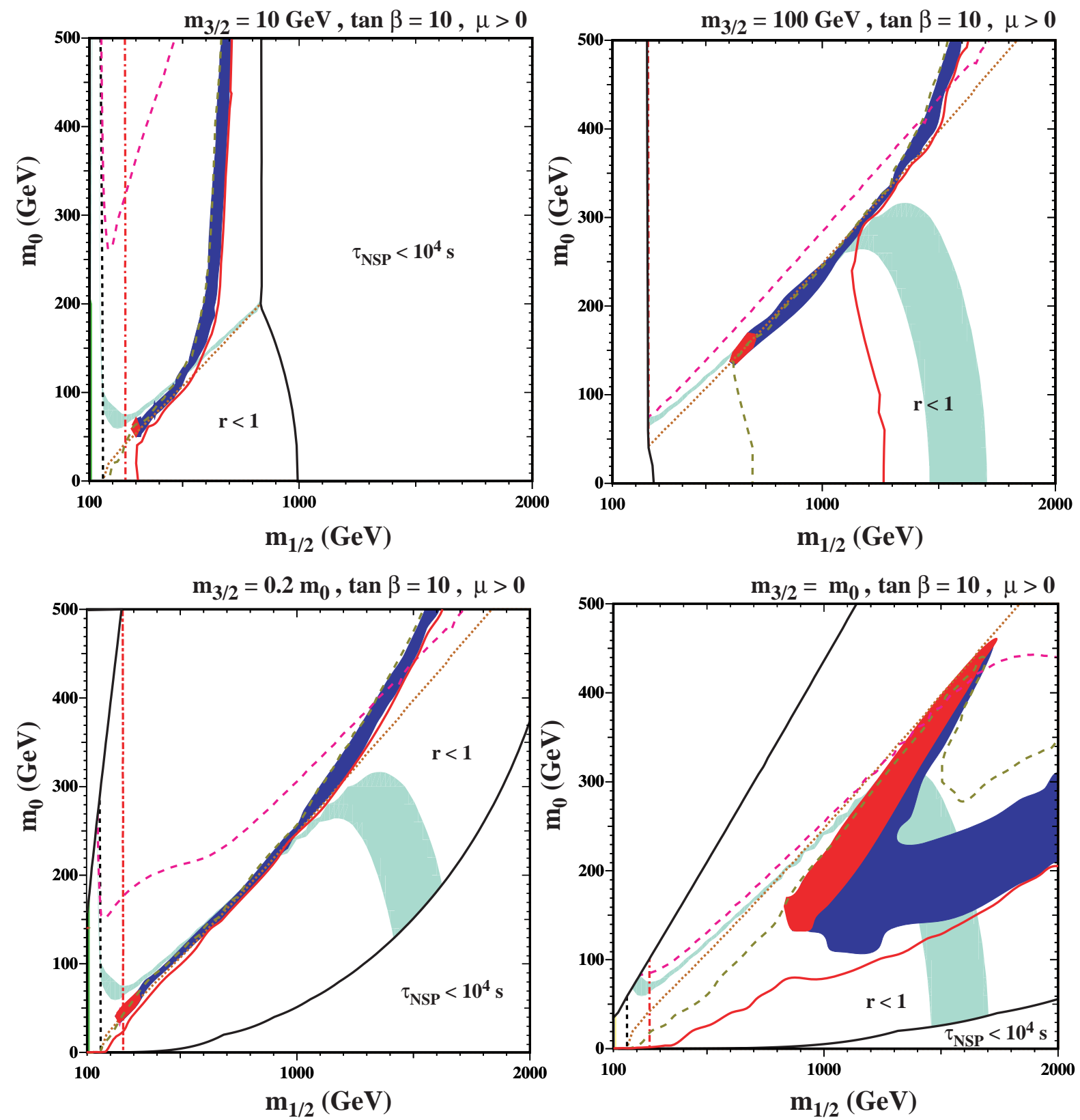

Figure 3: The $\left(m_{1 / 2}, m_{0}\right)$ planes for $\mu>0, \tan \beta=10$ and (a) $m_{3 / 2}=10 \mathrm{GeV}$, (b) $m_{3 / 2}=100 \mathrm{GeV}$, (c) $m_{3 / 2}=0.2 m_{0}$ and (d) $m_{3 / 2}=m_{0}$. We restrict our attention to the regions between the solid black lines, where the gravitino is the LSP and the NSP lifetime exceeds $10^{4} \mathrm{~s}$. In each panel, the near-vertical dashed black (dash-dotted red) line is the constraint $m_{\chi^{ \pm}}>104 \mathrm{GeV}\left(m_{h}>114 \mathrm{GeV}\right)$, the upper (purple) dashed line is the constraint $\Omega_{3 / 2} h^{2}<0.129$, and the light green shaded region is that where the NSP would have had $0.094 \leq \Omega h^{2} \leq 0.129$ if it had not decayed. The solid red (dashed grey-green) line is the region now (previously) allowed by the light-element abundances: $r<1$ as described in the text. The red (blue) shaded region is that where the ${ }^{7} \mathrm{Li}$ abundance could have been improved by NSP decays, but which is now excluded by the ${ }^{3} \mathrm{He}$ (D) constraint. 
the ranges shown in Fig. 2(a), and hence are suitable for modifying the ${ }^{7} \mathrm{Li}$ abundance, are shown as red and blue shaded regions in each panel of Fig. 3. We see that they straddle the erstwhile WMAP strips ${ }^{1}$. If we had been able to allow a Deuterium abundance as low as $\mathrm{D} / \mathrm{H} \sim(1-2) \times 10^{-5}$, the blue shaded region would have been able to resolve the $\mathrm{Li}$ discrepancy in the context of the CMSSM with gravitino dark matter. The blue region that we now regard as excluded by the lower limit on $\mathrm{D} / \mathrm{H}$, which is stronger than that used in [13], extends to large $m_{1 / 2}$. The red shaded region, which is consistent even with this limit on $\mathrm{D} / \mathrm{H}$, but yields very large ${ }^{3} \mathrm{He} / \mathrm{D}$, is close to the Higgs lower limit on $m_{1 / 2}$ for small $m_{3 / 2}$, moving to larger $m_{1 / 2}$ for larger $m_{3 / 2}$, so as to keep $\tau_{N S P}$ within the desired range.

We displayed in Fig. 1 the impact of the improved lower limit on $\mathrm{D} / \mathrm{H}$ and the new ${ }^{3} \mathrm{He}$ constraint on the abundance of an unstable particle, as a function of its lifetime. Interpreting this as a constraint on NSP decay into a gravitino, the panels in Fig. 3 show as solid red lines the additional restrictions these constraints impose on the $\left(m_{1 / 2}, m_{0}\right)$ planes for different values of $m_{3 / 2}$. The effects for small $m_{3 / 2}=10 \mathrm{GeV}$ [in panel (a)] $m_{3 / 2}=100 \mathrm{GeV}$ when $m_{0}$ is large [in panel (b)] and $0.2 m_{0}$ [in panel (c)] are relatively modest. This is because the limit occurs in a region where the NSP is a neutralino, and the relic density varies relatively rapidly. Hence a small change in the $m_{1 / 2}$ or $m_{0}$ results in a large change in $\zeta_{X}$, and the old and new bounds are relatively close. However, they do bite in the neighbourhood of the shaded ${ }^{7} \mathrm{Li}$ blobs, and have the effect of excluding them entirely. The effects for large $m_{3 / 2}=100 \mathrm{GeV}$ and small $m_{0}$ [in panel (b)] and $m_{3 / 2}=m_{0}$ [in panel (d)] are relatively large, mainly due to the slow variation of $\zeta_{X}$ near the limit which is characteristic of the $\tilde{\tau}$ NSP region, as can be seen from the relatively wide WMAP strips in this region. Reflecting this wide separation between the (old) dashed grey-green lines and the (new) solid red lines, we see large red and blue swaths in panel $(\mathrm{d})$, where the ${ }^{7} \mathrm{Li}$ abundance could have been reduced, but the ${ }^{3} \mathrm{He} / \mathrm{D}$ and/or $\mathrm{D} / \mathrm{H}$ ratios are unacceptable ${ }^{2}$.

Fig. 4 shows the corresponding $\left(m_{1 / 2}, m_{0}\right)$ planes for the choice $\tan \beta=57$, which is among the larger values allowed in the context of the constrained MSSM. Although the shapes of the allowed regions are rather different from the previous $\tan \beta=10$ case, the qualitative conclusions are similar. The ${ }^{3} \mathrm{He}$ constraint again has relatively modest impact. However, in all cases the red shaded ${ }^{7} \mathrm{Li}$ regions are excluded by the ${ }^{3} \mathrm{He}$ constraint, and the blue regions by the $\mathrm{D}$ abundance. We note that, in models with $m_{3 / 2}=m_{0}$ as in Fig. $4(\mathrm{~d})$, very little of the $\left(m_{1 / 2}, m_{0}\right)$ plane admits gravitino dark matter and, in the viable corner, there were no possibilities for depleting ${ }^{7} \mathrm{Li}$.

Finally, we consider very constrained models motivated by minimal supergravity (mSUGRA), in which not only is $m_{3 / 2}=m_{0}$, but also the trilinear soft supersymmetry-breaking parameter $A$ determines the bilinear soft supersymmetry-breaking Higgs-mixing parameter: $B=A-m_{3 / 2}$. This is compatible with the values of $\mu$ and $m_{A}$ specified by the electroweak vacuum conditions for only one value of $\tan \beta$ for any given pair of values of $\left(m_{1 / 2}, m_{0}\right)$ [17]. Such mSUGRA models are then specified by a choice of $A \equiv \hat{A} m_{3 / 2}$ : the panels of Fig. 5

\footnotetext{
${ }^{1}$ This raises the possibility that a discovery of supersymmetry might have an ambiguous interpretation neutralino LSP or gravitino LSP - in the absence of supplementary information.

${ }^{2}$ We note in passing that in several panels there are parts of the blue and/or red regions at large $m_{0}$ that are also excluded by the WMAP relic density limit.
} 

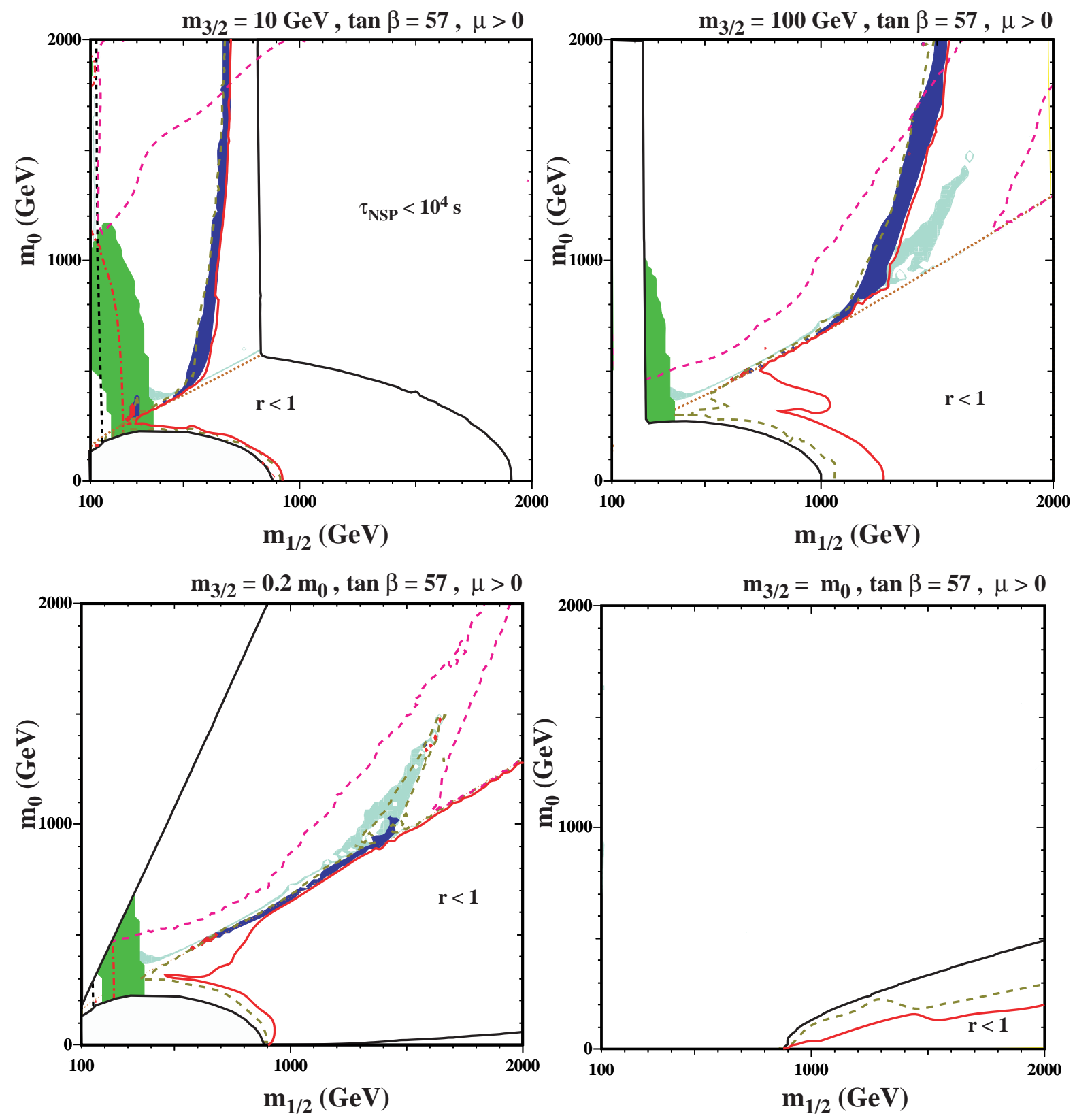

Figure 4: As in Fig. 3, but now for $\tan \beta=57$. 

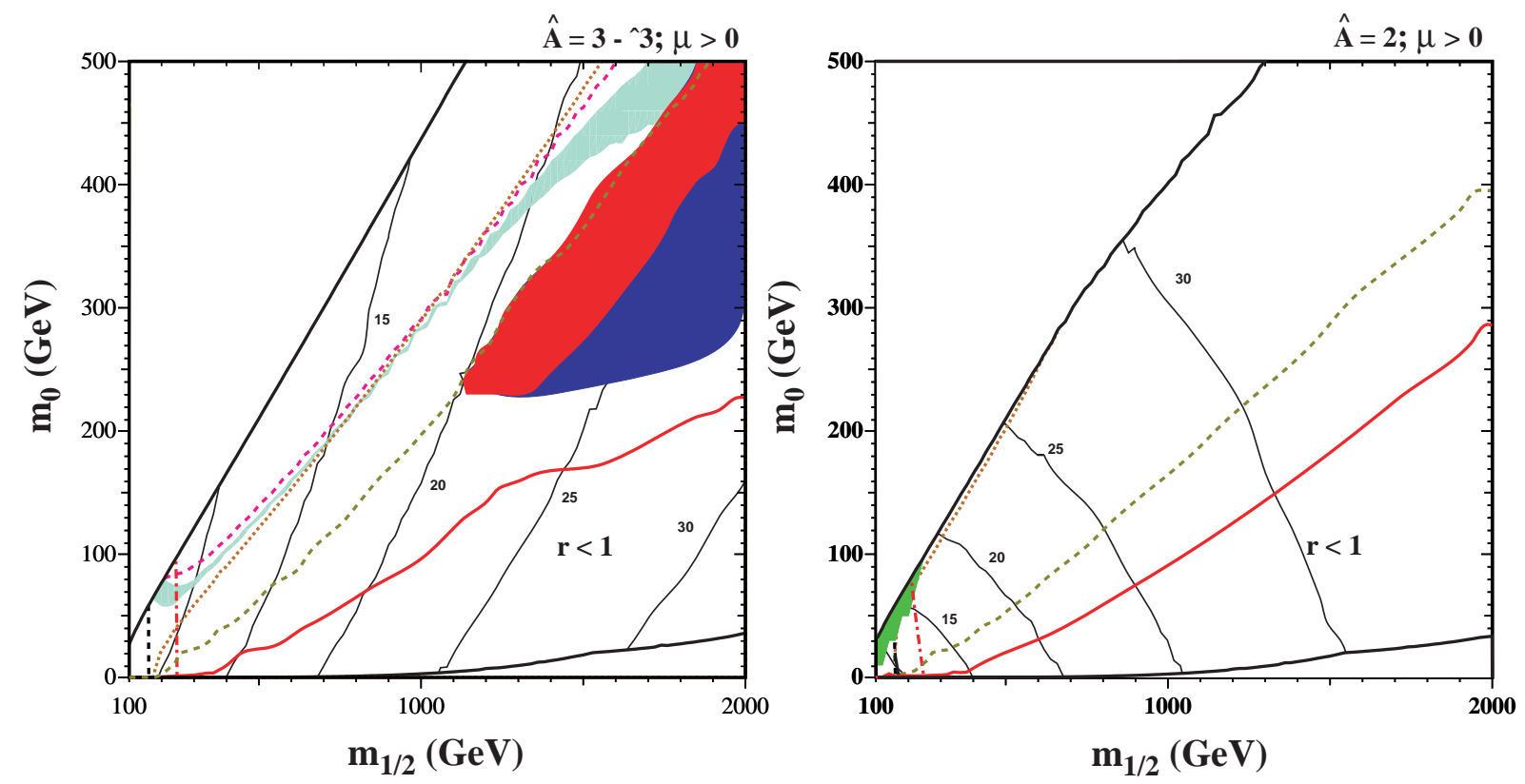

Figure 5: As in Fig. 3, but now for very constrained models motivated by mSUGRA. The value of $\tan \beta$ is fixed by the vacuum conditions, and varies across the $\left(m_{1 / 2}, m_{0}\right)$ planes with values indicated by the steep black contours. These models are specified by the choices of $A \equiv \hat{A} m_{3 / 2}$ : (a) $\hat{A}=(3-\sqrt{3})$, the Polonyi model, and (b) $\hat{A}=2$.

assume (a) $\hat{A}=(3-\sqrt{3})$ as found in the simple Polonyi model of supersymmetry breaking in mSUGRA [36], and (b) $\hat{A}=2$. For $\hat{A}=(3-\sqrt{3})$, the contours of constant $\tan \beta$ are approximately vertical, and range from about 10 at low $m_{1 / 2}$ to about 30 at high values of $m_{1 / 2}$. In the interesting region of panel (a) where ${ }^{7} \mathrm{Li}$ can be depleted, $\tan \beta \sim 20-30$. As before, we consider here only regions of the $\left(m_{1 / 2}, m_{0}\right)$ planes between the two solid black lines: above the higher one, the gravitino is no longer the LSP, and below the lower one the lifetime falls below $10^{4} \mathrm{~s}$. In addition to the constraints discussed earlier, panel (b) also displays a small green shaded region at low $m_{1 / 2}$ that is excluded by $b \rightarrow s \gamma$ decay.

We see that there is a large difference between the effects of implementing the old and new light-element constraints in panel (a) of Fig. 5 for $\hat{A}=(3-\sqrt{3})$, the Polonyi value, whereas the effect in panel (b) for $\hat{A}=2$ is smaller. In the Polonyi case, there are large ${ }^{7}$ Li-friendly regions that are excluded by the ${ }^{3} \mathrm{He}$ and $\mathrm{D}$ constraints. This reflects the fact that $\tan \beta$ is relatively small in this case, so the model is qualitatively similar to the $m_{3 / 2}=m_{0}$ case for $\tan \beta=10$ shown in panel (d) of Fig. 3. On the other hand, $\tan \beta$ is typically larger for $\hat{A}=2$, and when combined with a smaller stau mass, we find no visible ${ }^{7}$ Li-friendly region, as a result of small yet significant shifts in the values of both $\zeta_{X}$ and $\tau_{X}$.

\section{Conclusions}

In the absence of a convincing astrophysical explanation for the apparent discrepancy between the observed abundance of ${ }^{7} \mathrm{Li}$ and that calculated on the basis of the baryon-to-photon 
ratio inferred from CMB observations, it has been natural to explore the possible effects of late-decaying massive particles. Indeed, they could suppress the primordial abundance of ${ }^{7} \mathrm{Li}$, but at some price. Either the abundance of Deuterium should be very low compared with the latest available measurements, and/or the primordial ${ }^{3} \mathrm{He} / \mathrm{D}$ ratio must have been very high.

The latest observations of remote cosmological clouds along the lines of sight of highredshift quasars suggest that $\mathrm{D} / \mathrm{H}>2.2 \times 10^{-5}$, ruling out much of the parameter space for unstable particles that would otherwise have been suitable for diminishing the ${ }^{7} \mathrm{Li}$ abundance to agree with observations. A significant part of this parameter space would have been allowed by the more relaxed limit $\mathrm{D} / \mathrm{H}>1.3 \times 10^{-5}$ considered previously [13].

The remaining part of the parameter space for unstable particles that is consistent with the current lower limit would yield a ${ }^{3} \mathrm{He} / \mathrm{D}$ ratio at least an order of magnitude higher than the proto-solar value. Since $\mathrm{D}$ has been destroyed by stars, reducing its abundance from approximately 2.5 to $1.5 \times 10^{-5}$, while the ${ }^{3} \mathrm{He}$ abundance is thought to have remained roughly constant, a primordial ratio of ${ }^{3} \mathrm{He} / \mathrm{D}>1$ or 2 is unacceptable. This closes the remaining loophole for suppressing ${ }^{7} \mathrm{Li}$ without running into conflict with the other lightelement abundances.

We have also analyzed the potential bounds imposed by the ${ }^{6} \mathrm{Li}$ abundance. In principle, the high growth of its abundance between BBN and the formation of halo stars could also have been explained by the decays of the NSP in supersymmetric models, were it not for either the low resulting abundance of $\mathrm{D} / \mathrm{H}$ or the high ratio of ${ }^{3} \mathrm{He} / \mathrm{D}$.

So what is the interpretation of the apparent discrepancy between the calculations of the primordial ${ }^{7} \mathrm{Li}$ abundance and the Spite plateau? We have argued that the origin of the discrepancy cannot be the possible existence of unstable particles able to destroy the primordial nucleus. This leaves the problem open. Systematic uncertainties in nuclear effects such as higher ${ }^{7} \mathrm{Be}+\mathrm{D}$ reaction rates have been considered [6, 29], but seem unable to modify substantially the abundance of ${ }^{7} \mathrm{Li}$. Stellar mechanisms of depletion may be the last resort [26]. Perhaps other new and exciting astrophysical or physical effects will have to be considered.

A significant output of this analysis has been the demonstration of the importance of the ${ }^{3} \mathrm{He} / \mathrm{D}$ constraint on late-decaying massive particles as argued in [14]. Can one make the ${ }^{3} \mathrm{He}$ constraint more precise? This would require considering in more detail the cosmic evolution of D and ${ }^{3} \mathrm{He}$. One should allow for the possibility of exotic effects such as largescale destruction of this isotope in primitive structures such as massive Population-III stars, followed by moderate production by normal galactic evolution. However, any such scenario should consider simultaneously the cosmic evolution of D. As it is more fragile than ${ }^{3} \mathrm{He}, \mathrm{D}$ would also be destroyed in any Population-III stars. As we have seen, the ${ }^{3} \mathrm{He}$ constraint sharpens the embarrassment of supersymmetric models with heavy particles whose lifetimes are $\sim 10^{7}$ to $10^{8} \mathrm{~s}$. This interest motivates more detailed studies of the cosmic evolution of ${ }^{3} \mathrm{He}$ and $\mathrm{D}$, as well as ${ }^{6} \mathrm{Li}$ and ${ }^{7} \mathrm{Li}$.

Finally, it would be very useful if the observed abundances of $\mathrm{D} / \mathrm{H}$ in quasar absorption systems were improved. There is currently considerable dispersion in the observed abundances. We note that two such systems in the directions of Q2206-199 at $z=2.0762$ with 
$\mathrm{D} / \mathrm{H}=1.65 \pm 0.35 \times 10^{-5}[37]$ and PKS1937-1009 at $z=3.256$, with $\mathrm{D} / \mathrm{H}=1.6_{-0.30}^{+0.25} \times 10^{-5}[38]$ have quite low abundances of $\mathrm{D}$, similar to that observed at present in the ISM of our Galaxy [39]. If these measurements were to represent the correct $\mathrm{D} / \mathrm{H}$ abundance in those clouds, ${ }^{7} \mathrm{Li}$ depletion and ${ }^{6} \mathrm{Li}$ production by sparticle decay would be a viable option, though by solving one problem we would open two new problems. Why is the $\mathrm{D} / \mathrm{H}$ abundance in most of the other absorption systems significantly higher, and how can we account for the $\mathrm{D} / \mathrm{H}$ abundances in the solar system, which are also in the range $1.5-2.5 \times 10^{-5}$ ?

\section{Acknowledgments}

We thank R. Cyburt, B. Fields, Y. Santoso and V. Spanos for collaborations on related topics. The work of K.A.O. and E.V. was supported by the Project "CNRS/USA", and the work of K.A.O. was also supported partly by DOE grant DE-FG02-94ER-40823.

\section{References}

[1] T. P. Walker, G. Steigman, D. N. Schramm, K. A. Olive and K. Kang, Astrophys. J. 376 (1991) 51; K. A. Olive, G. Steigman, and T. P. Walker, Phys. Rep. 333 (2000) 389; B. D. Fields and S. Sarkar, Phys. Rev. D66 (2002) 010001.

[2] G. Steigman, D.N. Schramm, and J. Gunn, Phys. Lett. B66 (1977) 202; for a recent analysis see: R. H. Cyburt, B. D. Fields, K. A. Olive and E. Skillman, arXiv:astro$\mathrm{ph} / 0408033$.

[3] C. L. Bennett et al., Astrophys. J. Suppl. 148 (2003) 1 [arXiv:astro-ph/0302207]; D. N. Spergel et al., Astrophys. J. Suppl. 148 (2003) 175 [arXiv:astro-ph/0302209].

[4] R. H. Cyburt, B. D. Fields and K. A. Olive, Phys. Lett. B 567 (2003) 227 [arXiv:astro$\mathrm{ph} / 0302431]$.

[5] R. H. Cyburt, B. D. Fields and K. A. Olive, New Astron. 6 (1996) 215.

[6] A. Coc, E. Vangioni-Flam, P. Descouvemont, A. Adahchour and C. Angulo, Astrophys. J. 600 (2004) 544 [arXiv:astro-ph/0309480]

[7] P. Descouvemont, A. Adahchour, C. Angulo, A. Coc and E. Vangioni-Flam, ADNDT 88 (2004) 203 [arXiv:astro-ph/0407101].

[8] A. Cuoco, F. Iocco, G. Mangano, G. Miele, O. Pisanti and P. D. Serpico, Int. J. Mod. Phys. A 19 (2004) 4431 [arXiv:astro-ph/0307213].

[9] R. H. Cyburt, Phys. Rev. D 70 (2004) 023505 [arXiv:astro-ph/0401091].

[10] K. Jedamzik, Phys. Rev. D 70 (2004) 063524 [arXiv:astro-ph/0402344]; K. Jedamzik, Phys. Rev. D 70 (2004) 083510 [arXiv:astro-ph/0405583]. 
[11] J. L. Feng, A. Rajaraman and F. Takayama, Phys. Rev. D 68 (2003) 063504 [arXiv:hep$\mathrm{ph} / 0306024]$.

[12] D. Lindley, Astrophys. J. 294 (1985) 1; J. R. Ellis, D. V. Nanopoulos and S. Sarkar, Nucl. Phys. B 259 (1985) 175; D. Lindley, Phys. Lett. B 171 (1986) 235; M. H. Reno and D. Seckel, Phys. Rev. D 37 (1988) 3441; S. Dimopoulos, R. Esmailzadeh, L. J. Hall and G. D. Starkman, Nucl. Phys. B 311 (1989) 699; J. Ellis et al., Nucl. Phys. B 337 (1992) 399; M. Kawasaki and T. Moroi, Prog. Theor. Phys. 93 (1995) 879 [arXiv:hep-ph/9403364] and Astrophys. J. 452 (1995) 506 [arXiv:astro-ph/9412055]; E. Holtmann, M. Kawasaki, K. Kohri and T. Moroi, Phys. Rev. D 60 (1999) 023506 [arXiv:hep-ph/9805405]; M. Kawasaki, K. Kohri and T. Moroi, Phys. Rev. D 63 (2001) 103502 [arXiv:hep-ph/0012279]; K. Kohri, Phys. Rev. D 64 (2001) 043515 [arXiv:astro$\mathrm{ph} / 0103411]$.

[13] R. H. Cyburt, J. R. Ellis, B. D. Fields and K. A. Olive, Phys. Rev. D 67 (2003) 103521 [arXiv:astro-ph/0211258].

[14] M. Kawasaki, K. Kohri and T. Moroi, arXiv:astro-ph/0402490 and arXiv:astro$\mathrm{ph} / 0408426$.

[15] J. L. Feng, S. f. Su and F. Takayama, Phys. Rev. D 70 (2004) 063514 [arXiv:hepph/0404198]; J. L. Feng, S. Su and F. Takayama, Phys. Rev. D 70 (2004) 075019 [arXiv:hep-ph/0404231].

[16] J. R. Ellis, K. A. Olive, Y. Santoso and V. C. Spanos, Phys. Lett. B 588 (2004) 7 [arXiv:hep-ph/0312262].

[17] J. R. Ellis, K. A. Olive, Y. Santoso and V. C. Spanos, Phys. Lett. B 573 (2003) 162 [arXiv:hep-ph/0305212]; J. R. Ellis, K. A. Olive, Y. Santoso and V. C. Spanos, Phys. Rev. D 70 (2004) 055005 [arXiv:hep-ph/0405110].

[18] K. A. Olive and E. D. Skillman, Astrophys. J. 617 (2004) 29 [arXiv:astro-ph/0405588].

[19] D. Kirkman, D. Tytler, N. Suzuki, J. M. O’Meara and D. Lubin, Astrophys. J. Supp. 149 (2003) 1 [arXiv:astro-ph/0302006].

[20] S. G. Ryan, T. C. Beers, K. A. Olive, B. D. Fields, and J. E. Norris Astrophys. J. Lett. 530 (2000) L57 [ arXiv:astro-ph/9905211].

[21] M. Asplund et al. , (2004) in preparation.

[22] D. L. Lambert, AIP Conf. Proc. 743 (2005) 206 [arXiv:astro-ph/0410418].

[23] T. M. Bania, R. T. Rood, and D.S. Balser, Nature 415 (2002) 54.

[24] J. Geiss and G. Gloeckler, Sp. Sci. Rev. 84 (1998) 239; G. Gloeckler and J. Geiss, Sp. Sci. Rev. 84 (1998) 275. 
[25] C. P. Deliyannis, P. Demarque and S. D. Kawaler, Astrophys. J. Supp. 73 (1990) 21.

[26] S. Vauclair and C. Charbonnel, Astrophys. J. 502 (1998) 372; M. H. Pinsonneault, T. P. Walker, G. Steigman and V. K. Narayanan, Astrophys. J. 527 (1998) 180 [arXiv:astro-ph/9803073]; M. H. Pinsonneault, G. Steigman, T. P. Walker and V. K. Narayanan, Astrophys. J. 574 (2002) 398 arXiv:astro-ph/0105439; O. Richard, G. Michaud and J. Richer, A.A. 431 (2005) 1 [arXiv:astro-ph/0409672].

[27] S. G. Ryan, J. E. Norris and T. C. Beers, Astrophys. J. 523 (1999) 654 [ arXiv:astroph/9903059].

[28] J. Melendez and I. Ramirez, Astrophys. J. 615 (2004) L33 [arXiv:astro-ph/0409383].

[29] R. H. Cyburt, B. D. Fields and K. A. Olive, Phys. Rev. D 69 (2004) 123519 [arXiv:astro$\mathrm{ph} / 0312629]$.

[30] B. D. Fields and K. A. Olive, New Astronomy 4 (1999) 255; E. Vangioni-Flam, M. Cassé, R. Cayrel, J. Audouze, M. Spite and F. Spite, New Astronomy 4 (1999) 245.

[31] E. Rollinde, E. Vangioni-Flam and K. A. Olive, arXiv:astro-ph/0412426.

[32] E. Vangioni-Flam, K. A. Olive, B. D. Fields and M. Cassé, Astrophys. J. 585 (2003) 611 [arXiv:astro-ph/0207583].

[33] J. R. Ellis, T. Falk, G. Ganis, K. A. Olive and M. Srednicki, Phys. Lett. B 510 (2001) 236 [arXiv:hep-ph/0102098]; V. D. Barger and C. Kao, Phys. Lett. B 518 (2001) 117 [arXiv:hep-ph/0106189]; L. Roszkowski, R. Ruiz de Austri and T. Nihei, JHEP 0108 (2001) 024 [arXiv:hep-ph/0106334]; A. B. Lahanas and V. C. Spanos, Eur. Phys. J. C 23 (2002) 185 [arXiv:hep-ph/0106345]; A. Djouadi, M. Drees and J. L. Kneur, JHEP 0108 (2002) 055 [arXiv:hep-ph/0107316]; U. Chattopadhyay, A. Corsetti and P. Nath, Phys. Rev. D 66 (2002) 035003 [arXiv:hep-ph/0201001]; H. Baer, C. Balazs, A. Belyaev, J. K. Mizukoshi, X. Tata and Y. Wang, JHEP 0207 (2002) 050 [arXiv:hep-ph/0205325]; R. Arnowitt and B. Dutta, arXiv:hep-ph/0211417; J. R. Ellis, K. A. Olive, Y. Santoso and V. C. Spanos, Phys. Lett. B 573 (2003) 163 [arXiv:hep-ph/0308075].

[34] J. R. Ellis, K. A. Olive, Y. Santoso and V. C. Spanos, Phys. Lett. B 565 (2003) 176 [arXiv:hep-ph/0303043].

[35] H. Baer and C. Balazs, JCAP 0305 (2003) 006 [arXiv:hep-ph/0303114]; A. B. Lahanas and D. V. Nanopoulos, Phys. Lett. B 568 (2003) 55 [arXiv:hep-ph/0303130]; U. Chattopadhyay, A. Corsetti and P. Nath, Phys. Rev. D 68 (2003) 035005 [arXiv:hepph/0303201]; C. Munoz, arXiv:hep-ph/0309346; R. Arnowitt, B. Dutta and B. Hu, arXiv:hep-ph/0310103.

[36] J. Polonyi, Budapest preprint KFKI-1977-93 (1977); R. Barbieri, S. Ferrara and C.A. Savoy, Phys. Lett. 119B (1982) 343. 
[37] M. Pettini and D. V. Bowen, Astrophys. J. 560 (2001) 41 [arXiv:astro-ph/0104474].

[38] N. H. M. Crighton, J. K. Webb, A. Ortiz-Gill and A. Fernandez-Soto, MNRAS 355 (2004) 1042 [arXiv:astro-ph/0403512].

[39] H. W. Moos et al., Astrophys. J. Suppl. 140 (2002) 3 [arXiv:astro-ph/0112519]. 